\title{
Modeling Morphology and Catalytic Activity of Nanoparticle Ensembles Under Reaction Conditions
}

\author{
Raffaele Cheula, Matteo Maestri,* and Giannis Mpourmpakis*
}

Cite This: ACS Catal. 2020, 10, 6149-6158

Read Online

ABSTRACT: Although nanoparticle catalysts obtain different sizes and shapes under reaction conditions, computational modeling in heterogeneous catalysis is usually based on well-defined crystallographic planes. Herein, we combine density functional theory (DFT) calculations with Boltzmann statistics to describe ensembles of nanoparticles obtaining different morphologies under reaction conditions (temperature and gas-phase chemical potential) and their respective distribution of active sites. We apply our methodology on Rh catalytic nanoparticles, and we address the contribution of metastable nanostructures on the overall CO dissociation catalytic activity. Importantly, we demonstrate how catalytic trends can change when accounting for an ensemble of nanoparticles
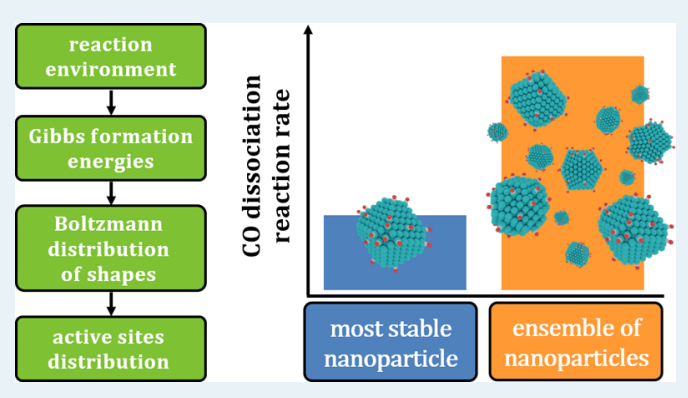
compared to a single, thermodynamically stable nanoparticle. Thus, our work enlightens the impact of statistical representation of catalyst morphology on modeling structure-sensitive reactions.

KEYWORDS: active sites, Boltzmann statistics, heterogeneous catalysis, nanoparticles, structure-activity relations

\section{INTRODUCTION}

Filling the materials gap in the modeling of heterogeneous catalysis is paramount in any effort toward the atomic-level mechanistic understanding of reaction mechanisms. ${ }^{1,2}$ Moreover, it is intrinsically required for the integration of firstprinciples calculations with the microkinetic modeling of catalytic reactions. In particular, the atoms at the surface of supported catalyst nanoparticles provide active sites with different catalytic activities depending on their structural arrangement and their coordination environment. This has been demonstrated by several theoretical studies on adsorption energies of reaction intermediates ${ }^{3,4}$ and activation energies of transition states. ${ }^{5-8}$ The identification and quantification of the catalytic active sites are of crucial importance in first-principles modeling of heterogeneous catalysis. This task heavily relies on the development of methods for the prediction of the catalyst structure under reaction conditions. Thus, it is of paramount importance to account for the dynamic character of the catalyst, since reaction intermediates can induce important morphological transformations. ${ }^{9-11}$

Toward this direction, Cheula et al. ${ }^{12}$ combined Wulff construction with microkinetic modeling and $a b$ initio thermodynamics to study the structure of $\mathrm{Rh}$ nanoparticles during the catalytic partial oxidation of methane. The Wulff construction is typically used in surface science to predict the equilibrium shape of single-crystal particles. ${ }^{13}$ When coupled with $a b$ initio thermodynamics, it can be used to calculate the equilibrium shape of nanoparticles as a function of the chemical potential of the surrounding gas phase reaction environment. ${ }^{14-17}$ As such, the morphological changes of the catalyst were captured in agreement with experimental observations, which are induced by the adsorption of reaction intermediates due to the variation of the gas phase chemical potential during reaction. ${ }^{12}$ The Wulff construction, however, provides only the ground-state (equilibrium) shape of the catalyst. Instead, experimental observations report that catalyst nanoparticles are not of the exact same morphology, but they are characterized by a significant diversity of shapes, ${ }^{18,19}$ which in turn, can affect the observed reactivity. For instance, at low reaction temperatures, nanoparticles can be "trapped" in metastable shapes because their evolution is hindered by slow formation kinetics. On the other hand, at high reaction temperatures, populations of shapes can be thermodynamically favored because the entropy associated with their multiplicity of states becomes significant. ${ }^{13,20}$ Moreover, catalyst samples can be also characterized by different bulk structures. In particular, face-centered cubic (fcc) metals can form nanometric icosahedral and decahedral nanoparticles characterized by a stretching of the internal bulk atomic positions. ${ }^{13}$ Such nanoparticles, called multiply-twinned particles (MTPs), are found along with fcc single crystals in catalyst samples, and their relative abundance changes with the variation of the surrounding gas phase chemical potential. In this situation, the

Received: February 28, 2020

Revised: April 16, 2020

Published: May 15, 2020 
Wulff construction can be an oversimplified model for the description of the catalyst structure under reaction. In fact, to develop realistic models for the catalyst morphology and the corresponding contribution to activity, it is necessary to take into account the diversity of shapes of catalyst nanoparticles under reaction conditions.

To this goal, we couple Boltzmann statistics with firstprinciples calculations to study heterogeneous catalytic systems under reaction conditions by explicitly accounting for the chemical potential of the gas environment and coverage at the catalyst surface. Boltzmann statistics has been applied in the literature to study ensembles of particle shapes. The groups of Alexandrova $^{21,22}$ and Sautet ${ }^{23}$ have investigated morphology and catalytic activity of ensembles of nanoclusters (particles with less than 100 atoms), which can be directly simulated by first-principles methods. Barnard et al. ${ }^{24}$ have studied particle ensembles in the large-size regime (particles bigger than 10 $\mathrm{nm}$ ), for which the continuum models provide good estimation of nanoparticle energy. The group of Erhart ${ }^{25}$ has applied Boltzmann statistics to describe nanoparticles in an intermediate-size regime $(1 \mathrm{~nm}<$ diameter $<10 \mathrm{~nm})$, which is of great importance for industrial applications. However, the latter work is limited to the study of nanoparticles at zero temperature and in vacuum, without accounting for the presence of a chemical environment. Thus, methodologies capturing the diversity of catalyst morphologies under reaction conditions and the impact on the overall reactivity for nanocatalyst sizes of industrial importance are vastly absent from the literature. This contribution addresses this gap by introducing a methodology to simulate catalytic activity of ensembles of nanoparticles under a chemical environment.

\section{COMPUTATIONAL DETAILS}

Electronic-structure calculations are performed using density functional theory (DFT), as implemented in the Quantum Espresso $^{26}$ suite of codes, with PBE-GGA ${ }^{27}$ ultrasoft pseudopotentials and a plane wave basis set. The plane wave and electronic density cutoff energies are set to 35 and $280 \mathrm{Ry}$, respectively. A Monkhorst-Pack ${ }^{28}$ grid of $12 \times 12 \times 12 k$ point sampling is employed for bulk $\mathrm{Rh}$ and a proportional grid is used to ensure an equivalent sampling of reciprocal space for supercells. A Marzari-Vanderbilt cold smearing ${ }^{29}$ of $10^{-3} \mathrm{Ry}$ is applied. Periodic slabs with inversion symmetry are used to represent Rh crystal facets. A slab height higher than $10 \AA$ is used and the first three layers of the slabs are allowed to relax. Gas phase and cluster calculations are performed in cubic supercells. Vacuum (10 $\AA$ ) separates the atoms of neighboring supercells. For nanoclusters, all of the atoms are allowed to relax. The climbing-image nudged elastic band $\left(\mathrm{CI}-\mathrm{NEB}^{30}\right.$ ) method is used to identify the transition states of the elementary steps, with a 10 image path sampling and a final force convergence threshold of $0.05 \mathrm{Ry} / \AA \AA$. Vibration analysis has been performed with the finite-difference method, as implemented in the atomic simulation environment (ASE) library. ${ }^{31}$ For normal frequency evaluation, a displacement of $0.01 \AA$ was applied to all of the atoms allowed to relax in the corresponding electronic-structure calculations.

\section{RESULTS AND DISCUSSION}

To apply Boltzmann statistics in modeling of the catalyst morphology under realistic reaction conditions, an ensemble of nanoparticles, which represents the gamut of shapes of catalyst samples, is required. The ensemble should contain the most stable structures as well as metastable structures that can have lower but non-negligible frequency of occurrence in the distribution. Here, we employ a two-step procedure to obtain such an ensemble. The procedure is implemented in a homemade library of Python and Cython ${ }^{32}$ scripts, which uses tools of the atomic simulation environment (ASE) library. $^{31}$ In the first step, we create an ensemble of nanoparticles that expose complete crystal facets along with edges and kinks at their boundaries. We include in the ensemble different nanoparticles that have been experimentally observed (i.e., single crystals and decahedra and icosahedra structures). ${ }^{13}$ For each nanoparticle type, we cleave the bulk phase with a set of planes drawn at different distances from a central point. The set comprises low- and high-index Miller planes. In Figure 1, we demonstrate three examples of

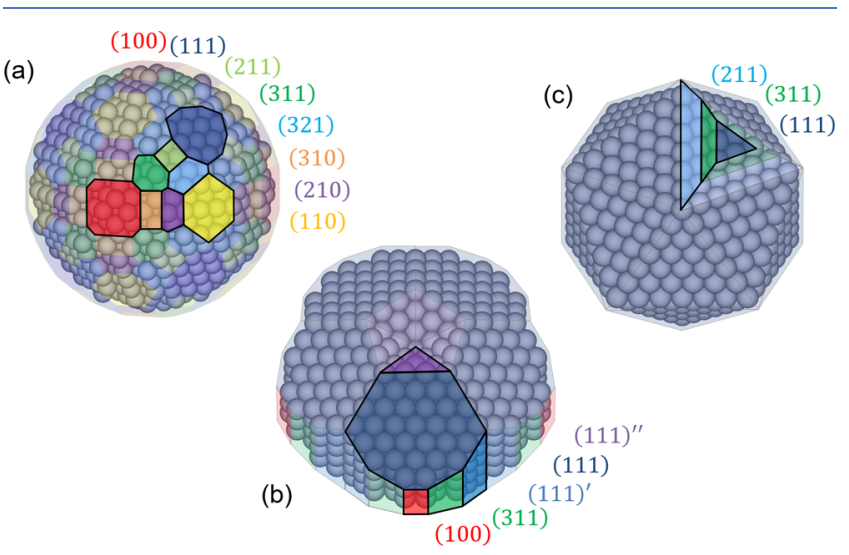

Figure 1. Planes considered for creating the ensemble of nanoparticle shapes for fcc particles (a), decahedra (b), and icosahedra (c). For fcc particles, the planes are named with their Miller indices, whereas for MTPs, the names of the planes are assigned based on the surface structure that they create. For decahedra, (111) is the plane that cut the top and bottom surfaces of the particle creating (111)-like surfaces; the planes $(111)^{\prime},(100)$, and (311) cut the lateral surfaces of the particle and the plane (111)" creates the hole at the top and bottom of the particle. For icosahedra, each subunit is cut with (111), (211), and (311) planes, which create islands of atoms on (111)-like surfaces with different dimensions.

nanoparticles with the planes employed in the procedure. Each plane is created at a distance multiple of the interlayer distance of the corresponding crystal facet. The planes that belong to the same symmetry-related groups (e.g., (100) and (010) planes of fcc particles) are drawn at the same distance or at different distances (up to one layer). In this way, we produce both high- and low-symmetry nanoparticle shapes. All combinations of plane distances below a distance threshold are considered.

The resulting ensemble, however, is not continuous with respect to the number of atoms in the nanoparticles. Therefore, in the second step of the procedure, we create additional nanoparticles exposing incomplete crystal facets from the initial ensemble. As such, for a given nanoparticle in the initial ensemble with $\mathrm{N}$ atoms, one atom is removed, thus obtaining a new structure with $(\mathrm{N}-1)$ atoms. If this new structure does not already exist in the ensemble, then it is added, and the procedure continues by removing another atom. When the resulting structure is already present in the ensemble, the procedure is terminated. The same process is 
repeated on the whole set of nanoparticles present in the initial ensemble. The atoms removed are the ones with the lowest coordination number (exhibiting lowest cohesion), and when multiple atoms have the same coordination number, the sum of the coordination number of the neighbors is compared and the atom with the lowest one is removed. In this way, we iteratively and consistently remove the least stable atoms. ${ }^{33,34}$ With such a procedure, we produce an ensemble of more than 150000 nanoparticles with the number of atoms lower than 1200. The resulting ensemble contains nanoparticles with different bulk structures and different exposed crystal facets, and it is continuous in the number of atoms. More details on the construction of the ensemble of structures are reported in the Supporting Information (Section 1).

The application of Boltzmann distribution necessitates to calculate the energy of each nanoparticle of the ensemble. To this purpose, the use of first-principles calculations such as DFT is extremely computationally demanding and becomes intractable in computational cost for nanoparticles with number of atoms larger than a couple of hundred. To overcome this issue, in this work, we develop a model for the calculation of the energy of $\mathrm{Rh}$ nanoparticles with any arbitrary shape and size and we apply it for the energy calculation of the nanoparticles in the ensemble. The model is trained on a set of DFT calculations and includes also the effect of gas phase chemical potential and surface coverage on the nanoparticle energy.

According to the tight-binding theory, the formation energy $E_{\mathrm{f}, i}^{0}$ of one atom in a metallic nanoparticle is proportional to the square root of its coordination number, $\mathrm{CN}_{i}$, as described in the square-root bond cutting model ${ }^{33,34}$

$$
E_{\mathrm{f}, i}^{0}\left(\mathrm{CN}_{i}\right)=E_{\mathrm{Rh}, \mathrm{coh}}^{\text {bulk }} \sqrt{\mathrm{CN}_{i}} / \sqrt{12}-\left(E_{\mathrm{Rh}}^{\text {bulk }}-E_{\mathrm{Rh}}^{\mathrm{gas}}\right)
$$

Here, in parenthesis, we have changed our reference from the energy of one isolated $\mathrm{Rh}$ atom, $E_{\mathrm{Rh}}^{\text {gas }}$, to the energy of one $\mathrm{Rh}$ atom in the fcc bulk, $E_{\mathrm{Rh}}^{\text {bulk} . ~ T h e ~ f o r m a t i o n ~ e n e r g i e s ~ a r e ~}$ therefore calculated with respect to the bulk phase. $E_{\mathrm{Rh} \text {,coh }}^{\mathrm{bulk}}$ the metal cohesive energy (binding energy per atom) in the fcc bulk phase. The effect of the relaxation of the atoms at the surface is then included in the model. ${ }^{35}$ Moreover, to represent MTPs, additional terms are added to capture the effect of the twin boundaries, which separate their different subunits, and of the internal strain induced by the stretching of their bulk phase. $^{36}$ These effects can be considered negligible when comparing the energy of nanoparticles of different materials ${ }^{34}$ but not when we compare different shapes of the same material. The formation energy of the nanoparticles, $E_{\mathrm{f}}^{\mathrm{NP}}$, is calculated therefore as

$$
E_{\mathrm{f}}^{\mathrm{NP}}=\sum_{i}^{N_{\mathrm{Rh}}}\left[E_{\mathrm{f}, i}^{0}\left(\mathrm{CN}_{i}\right)+E_{\mathrm{relax}, i}\left(\mathrm{CN}_{i}\right)\right]+N_{\mathrm{tw}} E_{\mathrm{tw}}+N_{\mathrm{Rh}} E_{\mathrm{str}}
$$

where $N_{\mathrm{Rh}}$ is the total number of Rh atoms, $N_{\mathrm{tw}}$ is the number of atoms at the twin boundaries of MTPs, and $E_{\mathrm{tw}}$ is the twin boundary energy per atom calculated with DFT. $E_{\text {str }}$ is the strain energy per atom calculated with the analytical solution proposed by Howie and Marks. ${ }^{36} E_{\text {relax }}$ is the relaxation energy of one atom at the surface obtained by fitting DFT relaxation energies with the one-parameter formula proposed by Sun et al. $^{35}$

$$
E_{\text {relax }, i}\left(\mathrm{CN}_{i}\right)=\frac{E_{\mathrm{Rh}, \mathrm{coh}}^{\text {bulk }}}{12}\left\{\frac{2}{1+\exp \left[\frac{\left(12-\mathrm{CN}_{i}\right)}{8 \mathrm{CN}_{i}}\right]}\right\}^{-m}
$$

where $m=2.68$. The $E_{\text {relax }}$ results are shown in Figure $S 1$. Details on the values of the parameters and the models adopted to calculate them are reported in the Supporting Information (Section 2).

In the presence of adsorbates, the model is extended by accounting for the effect of their binding on the formation energy of the nanoparticles. As an example, here, we consider the effect of $\mathrm{CO}$ coverage, which has been reported as the most abundant reaction intermediate (MARI) of different reacting systems on $\mathrm{Rh}$, such as $\mathrm{CH}_{4}$ steam reforming, methanation, CO oxidation, and water-gas shift. ${ }^{37,38}$ Moreover, CO has been reported to be the major species responsible for the modifications of the catalyst surface morphology, and thus it is expected to substantially impact the distribution of active sites under reaction conditions. ${ }^{12}$ The formation energy of a nanoparticle in the presence of adsorbed $\mathrm{CO}$ molecules, $E_{\mathrm{f}}^{\mathrm{NP}+\mathrm{CO} *}$, is calculated as

$$
E_{\mathrm{f}}^{\mathrm{NP}+\mathrm{CO}^{*}}=E_{\mathrm{f}}^{\mathrm{NP}}+\sum_{i}^{N_{\mathrm{CO} *}} E_{\mathrm{bind}, i}^{\mathrm{CO} *}
$$

where $N_{\mathrm{CO}}$ is the number of adsorbed molecules and $E_{\mathrm{bind}, i}^{\mathrm{CO} *}$ is the $\mathrm{CO}$ binding energy, which is calculated as the sum of metal-adsorbate interactions and adsorbate-adsorbate interactions. The metal-adsorbate interaction is represented by the binding energy of $\mathrm{CO}$ at zero coverage, $E_{\mathrm{bind}, i}^{0, \mathrm{CO}} *$. The adsorbate-adsorbate interaction is the modification of the binding energy induced by lateral interactions between adsorbates, $\Delta E_{\text {bind }}^{\mathrm{CO} *}$.

To provide a model for $E_{\text {bind, } i}^{0, \mathrm{CO}_{*}}$, we analyze the adsorption of $\mathrm{CO}$ on top of $\mathrm{Rh}$ atoms with different coordination numbers, as the experimental observations report that at low coverage, $\mathrm{CO}$ adsorbs on the top sites of Rh facets. ${ }^{39-43}$ Then, to represent $\Delta E_{\text {bind }}^{\mathrm{CO}}$, we study the adsorption of $\mathrm{CO}$ on $\mathrm{Rh}$ crystal facets at high coverage, considering also the adsorption on sites other than the top sites and the formation of ordered patterns of $\mathrm{CO}^{*}$ on $\mathrm{Rh}$ that are observed in experiments. ${ }^{39,40,42,43}$ As the PBE functional is known to overestimate the binding energies of $\mathrm{CO}$ on $\mathrm{Rh}$ surfaces, we use the correlation proposed by Mason et $\mathrm{al}^{44}$ to correct the adsorption calculations.

The $\mathrm{CO}^{*}$ binding energy at zero coverage $\left(E_{\mathrm{bind}, i}^{0, \mathrm{CO}_{*}}\right)$ is linearly related to the coordination number of the $\mathrm{Rh}$ atom, at which it is adsorbed on the top configuration (results shown in Figure S4)

$$
E_{\text {bind, } i}^{0, \mathrm{CO}^{*}}\left(\mathrm{CN}_{i}\right)=a+b \mathrm{CN}_{i}
$$

where $a=-1.836$ and $b=3.494 \times 10^{-2}$. At high coverage, our calculations show that $\mathrm{CO}^{*}$ molecules relax their positions and tilt their adsorption angles to maximize their distances, resulting in distorted centered-hexagonal patterns (represented in Figure S5). As $\mathrm{CO}^{*}$ tends to occupy all of the available surface area on the Rh crystal facets, we express the effect of lateral interactions, $\Delta E_{\text {bind }}^{\mathrm{CO}}$, as a function of the ratio between the total surface area of the systems $\left(S_{\text {tot }}\right)$ and the total number of adsorbed $\mathrm{CO}$ molecules $\left(N_{\mathrm{CO}}\right)$. We use a power law to describe such a correlation (shown in Figure S6) 


$$
\Delta E_{\text {bind }}^{\mathrm{CO} *}\left(S_{\text {tot }} / N_{\mathrm{CO} *}\right)=\alpha\left(S_{\text {tot }} / N_{\mathrm{CO} *}\right)^{-\beta}
$$

where $\alpha=3.034 \times 10^{+2}$ and $\beta=3.31$. The CO binding energy is calculated therefore as

$$
\begin{aligned}
& E_{\text {bind }, i}^{\mathrm{CO} *}\left(\mathrm{CN}_{i}, S_{\text {tot }} / N_{\mathrm{CO} *}\right) \\
& \quad=E_{\text {bind }, i}^{0, \mathrm{CO}^{*}}\left(\mathrm{CN}_{i}\right)+\Delta E_{\text {bind }}^{\mathrm{CO} *}\left(S_{\text {tot }} / N_{\mathrm{CO} *}\right)
\end{aligned}
$$

The effect of the change in adsorption sites observed at high coverages is included in the formulation of $\Delta E_{\text {bind }}^{\mathrm{CO} *}$, as the effect of lateral interactions is always higher than the difference in binding energies between the new occupied sites and the top sites. Details on the values of the parameters and the models adopted to calculate them are reported in the Supporting Information (Section 2).

All of the parameters of the model are obtained with periodic DFT calculations. $E_{\text {twin }}$ and $E_{\text {strain }}$ are obtained with supercells periodic in the three dimensions. Slab models are used to calculate the $m$ parameter (eq 3) of $E_{\text {relax }, i}\left(\mathrm{CN}_{i}\right)$ and the four parameters ( $a$ and $b$ in eq $5, \alpha$ and $\beta$ in eq 6) of $E_{\text {bind }, i}^{\mathrm{CO} *}\left(\mathrm{CN}_{i}, S_{\text {tot }} / N_{\mathrm{CO}^{*}}\right)$. Then, the model is tested on reproducing the DFT energy of a set of new different structures including nanowires and nanoclusters, computationally much more demanding. For nanoparticles, $S_{\text {tot }}$ is calculated with the following procedure. First, we create a convex hull connecting all of the surface atoms and we draw a 3D surface that contains all atoms. Then, we enlarge such a surface by increasing the distance of each of its point from the origin by the average bond length of $\mathrm{CO}^{*}$ on Rh facets $(1.2 \AA)$ and we calculate the area of the resulting surface. We use this area for the evaluation of the effect of lateral interactions between $\mathrm{CO}^{*}$ molecules on nanoparticles (with eq 6).

In Figure 2, the parity plots between the formation energy calculated with DFT and the ones calculated with the model are presented. In panel (a), the model is tested on clean slabs, wires, and nanoclusters. In panel (b), the model is tested on slabs and nanoclusters at low and high CO* coverages. A good agreement between the model and the DFT calculations is found: the differences between the energies obtained with the model and DFT calculations are generally lower than $0.04 \mathrm{eV} /$ atom. The only outlier is the clean icosahedral $\mathrm{Rh}_{55}$. The average error introduced by the model is comparable to that of the DFT functionals employed in the state-of-the-art modeling of metallic surfaces and nanoclusters. The data sets of all of the DFT calculations results employed to train and validate the model are reported in Tables S1-S3.

To account for the effect of the experimental conditions on the energy of the nanoparticles, we introduce enthalpic and entropic contributions in the model. To this aim, vibrational analysis on surface structures is carried out. The effect of the pressure on the Gibbs free energy of solid structures is neglected in the analysis. ${ }^{45}$ In analogy with eq 4 , the formation Gibbs free energy of a nanoparticle in the presence of $\mathrm{CO}^{*}$ adsorbed molecules, $G_{\mathrm{f}}^{\mathrm{NP}+\mathrm{CO} *}$, is calculated as

$$
G_{\mathrm{f}}^{\mathrm{NP}+\mathrm{CO}^{*}}=G_{\mathrm{f}}^{\mathrm{NP}}+\sum_{i}^{N_{\mathrm{CO} *}} G_{\text {bind }, i}^{\mathrm{CO} *}
$$

The formation Gibbs free energy clean nanoparticles, $G_{\mathrm{f}}^{\mathrm{NP}}$, is calculated as

$$
G_{\mathrm{f}}^{\mathrm{NP}}=E_{\mathrm{f}}^{\mathrm{NP}}+\sum_{i}^{N_{\mathrm{Rh}}} \Delta G_{i}^{\mathrm{Rh}}\left(\mathrm{CN}_{i}, T\right)
$$
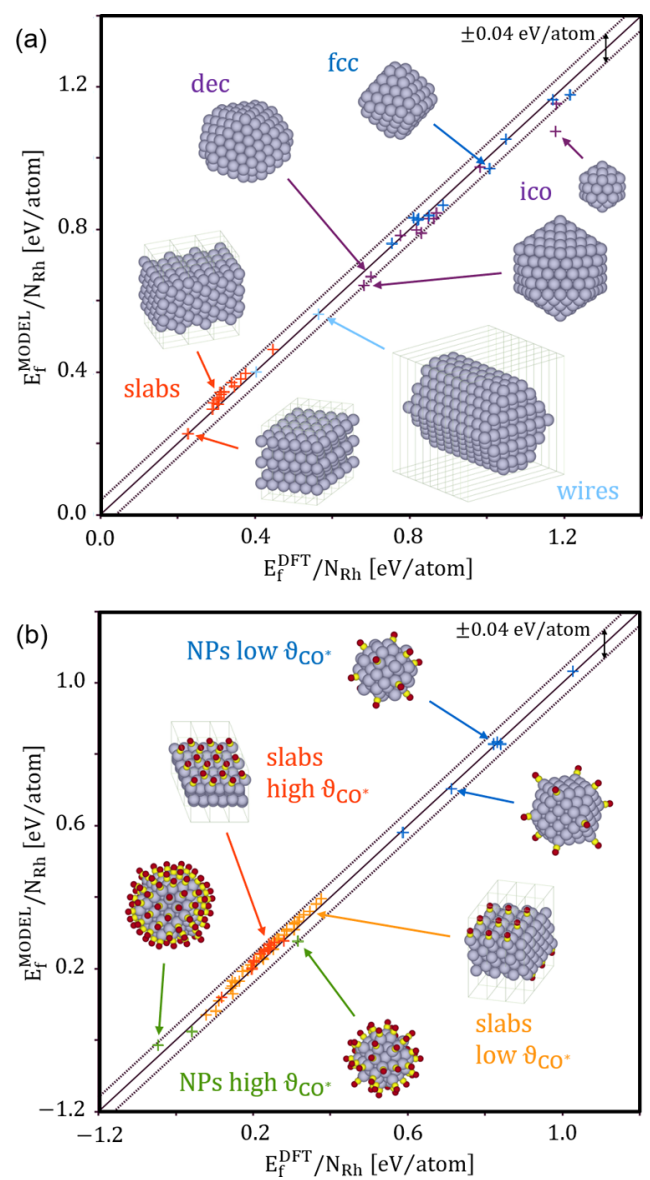

Figure 2. Parity plots of formation energy (per Rh atom) calculated with the model described in this work and with DFT. The references for the calculation of the formation energies are set to the fcc Rh bulk and isolated CO molecules. In (a), the model is tested on slabs, wires, and clusters with fcc, decahedral, and icosahedral bulk structure in the absence of adsorbed CO. In (b), the model is tested on slabs and clusters with low and high $\mathrm{CO}$ coverages.

where $\Delta G_{i}^{\mathrm{Rh}}$ is the Gibbs vibrational free energy of surface atoms calculated using the harmonic oscillator approximation. The binding Gibbs free energy of $\mathrm{CO}^{*}$ molecules, $G_{\mathrm{bind}, i}^{\mathrm{CO} *}$ is calculated as

$$
G_{\mathrm{bind}, i}^{\mathrm{CO} *}=E_{\mathrm{bind}, i}^{\mathrm{CO} *}+\Delta G_{i}^{\mathrm{CO} *}\left(\mathrm{CN}_{i}, T\right)-\Delta \mu_{\mathrm{gas}}^{\mathrm{CO}}\left(T, P_{\mathrm{CO}}\right)
$$

where $\Delta G_{i}^{\mathrm{CO} *}$ contains the vibrational and translational free energy of adsorbed $\mathrm{CO} *$ molecules. To represent $\mathrm{CO} *$ at both low and high temperatures, the hindered translator model ${ }^{46}$ has been adopted. As in the case of the binding energy, a linear correlation with the coordination number is found for $\Delta G_{i}^{\mathrm{CO} *}$

$$
\Delta G_{i}^{\mathrm{CO} *}\left(\mathrm{CN}_{i}\right)=c+d \mathrm{CN}_{i}
$$

where $c=-0.869$ and $d=2.770 \times 10^{-2} . \Delta \mu_{\text {gas }}^{\mathrm{CO}}$ contains the vibrational, rotational, and translational energy of $\mathrm{CO}$ in the gas phase evaluated using NASA coefficients ${ }^{47}$ based on experimental data. The pressure term in $\Delta \mu_{\text {gas }}^{\mathrm{CO}}$ is calculated in the ideal gas approximation

$$
\Delta \mu_{\text {gas }}^{\mathrm{CO}}\left(T, P_{\mathrm{CO}}\right)=\Delta \mu_{\text {gas }}^{0, \mathrm{CO}}(T)+k_{\mathrm{B}} T \ln \left(P_{\mathrm{CO}} / P_{0}\right)
$$

where $P_{0}$ is the reference pressure equal to $1 \mathrm{~atm}$. Details on the values of the parameters and the models adopted to 
calculate them are reported in the Supporting Information (Section 2).

For each particle, the $\mathrm{CO}^{*}$ coverage at the thermodynamic equilibrium between the adsorbates and the gas phase is calculated by minimizing the Gibbs formation energy of the nanoparticle with respect to the number of $\mathrm{CO}^{*}$ molecules. To this aim, we increase the total number of adsorbed $\mathrm{CO}^{*}$ molecules, $N_{\text {tot }}^{\mathrm{CO} *}$, from zero to the total number of surface atoms (with coordination number up to 9), and at each step, we calculate the corresponding formation Gibbs free energy of the nanoparticle using eq 8. For each value of $N_{\text {tot }}^{\mathrm{CO} *}$, the distribution of the $\mathrm{CO}^{*}$ molecules on the different adsorption sites is calculated by imposing thermodynamic equilibrium between the sites. Therefore, for each couple of adsorption sites, $i$ and $k$, with different coordination numbers we have

$$
\begin{aligned}
& \frac{\vartheta_{i}^{\mathrm{CO}}}{\vartheta_{k}^{\mathrm{CO}}}=\exp \left(-\frac{G_{\text {bind }, i}^{\mathrm{CO}^{*}}-G_{\text {bind }, k}^{\mathrm{CO}^{*}}}{k_{\mathrm{B}} T}\right) \\
& \sum_{i}^{9}\left(\vartheta_{i}^{\mathrm{CO}^{*}} N_{i}\right)=N_{\mathrm{tot}}^{\mathrm{CO}}
\end{aligned}
$$

where $N_{i}$ and $N_{k}$ are the number of surface atoms with coordination number $\mathrm{CN}_{i}$ and $\mathrm{CN}_{k}$, respectively, and $\vartheta_{\mathrm{i}}^{\mathrm{CO} *}$ and $\vartheta_{\mathrm{k}}^{\mathrm{CO}} *$ are their corresponding coverages. $k_{\mathrm{B}}$ is the Boltzmann constant. By repeating the calculation for each value of $N_{\text {tot }}^{\mathrm{CO} *}$, we obtain a function that describes how the Gibbs formation energy of the nanoparticle changes with the number of adsorbed $\mathrm{CO}^{*}$. The equilibrium coverage corresponds to the minimum of such a function. The different operating conditions (given in terms of different $\mathrm{CO}$ partial pressures in Figure 3a) lead to different values of $\mathrm{CO}$ equilibrium coverage. Moreover, at the same operating conditions, nanoparticles with different shapes can have different coverages, as shown in Figure 3b.

The model is applied for the calculation of the Gibbs formation energy of the whole ensemble of nanoparticles, as a function of temperature and $\mathrm{CO}$ partial pressure. Figure $4 \mathrm{a}$ shows the Gibbs formation energy per metal atom of the ensemble at $823 \mathrm{~K}$ and in the absence of $\mathrm{CO}$ in the gas phase. In agreement with previous analyses, ${ }^{25,48}$ we find that at different nanoparticle sizes (number of atoms), different types of nanoparticles form the most stable structures (lower in Gibbs formation energy). Two high-symmetry icosahedral nanoparticles are the most stable at very small sizes (55 and 147 atoms). By increasing the number of atoms, decahedra and fcc nanoparticles compete to be the most stable, and for larger number of atoms ( $>380$ atoms), fcc single crystal structures exhibit the lowest Gibbs formation energy. The formation energy of icosahedral ground state is described by jumps between nanoparticles with complete shells and high levels of symmetry.

Once we produced the ensemble of nanoparticles and calculated their energy, we analyzed their relative amount as a function of the operating conditions with a Boltzmann distribution. We do not limit our study to a fixed number of atoms, as catalyst samples generally show a distribution of particle sizes. A Boltzmann distribution of nanoparticles with the same number of atoms is independent of the reference energy of the metal because when we compare their formation energies, reference energies of metal atoms cancel out. The situation is different when we consider nanoparticles with a
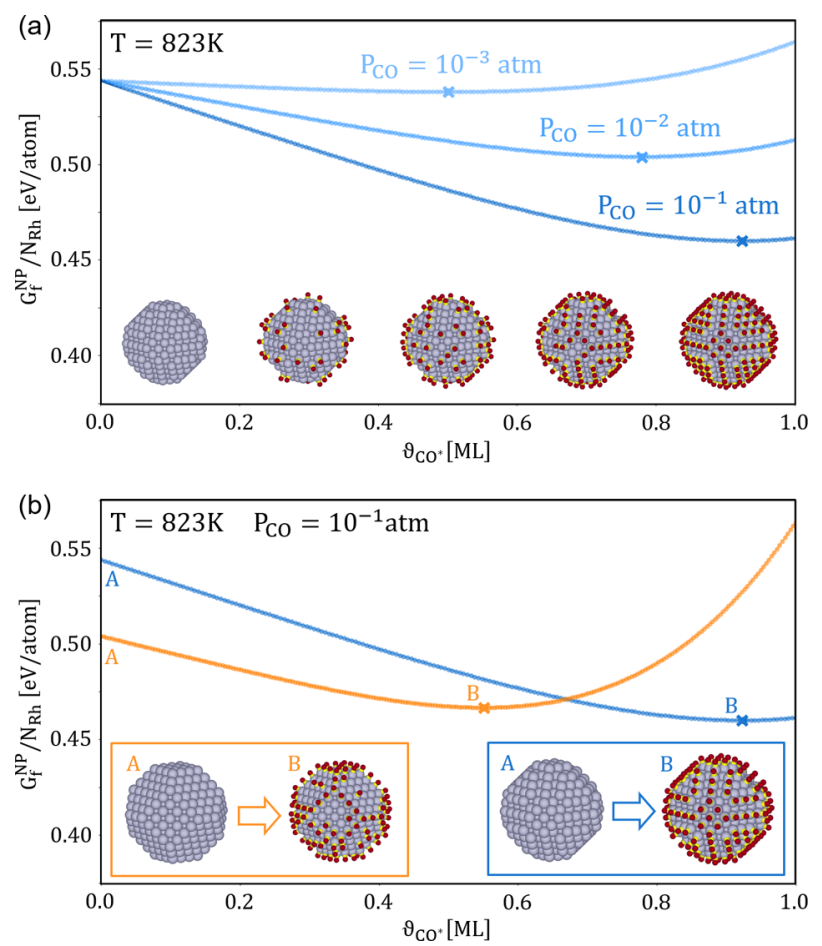

Figure 3. (a) Gibbs formation energy (per Rh atom) of a nanoparticle made of 550 atoms as a function of the $\mathrm{CO}^{*}$ coverage at $823 \mathrm{~K}$ and different partial pressures of $\mathrm{CO}$ in the gas phase. (b) Comparison between the Gibbs formation energy functions of two different nanoparticles with 550 atoms at $823 \mathrm{~K}$ and $P_{\mathrm{CO}}=0.1 \mathrm{~atm}$. The facetted nanoparticle (on the left) is more stable in the absence of CO (A), whereas the spherical one (on the right) becomes more stable in the presence of $\mathrm{CO}(\mathrm{B})$.

different number of atoms. In such a case, the results are dependent on the reference system used in the calculation of the formation energies. Hence, it is necessary to use as reference system the actual reservoir of metal atoms at the experimental conditions. ${ }^{49}$ The Gibbs free energies of formation calculated with eqs $1-14$ use as references the electronic energy of $\mathrm{Rh}$ in the fcc bulk phase and the chemical potential of $\mathrm{CO}$ molecules at the experimental conditions. Therefore, for each $\mathrm{Rh}$ atom in the nanoparticles, we need to change its reference energy from $E_{\mathrm{Rh}}^{\text {bulk }}($ eq 1$)$ to the chemical potential of $\mathrm{Rh}$ atoms $\left(\mu_{\mathrm{Rh}}\right)$ of the reservoir available in the system. As a result, the Boltzmann distribution, which describes the probability $p_{i}$ of finding each nanoparticle $i$ present in the ensemble, is calculated with the following formula

$$
p_{i}=\frac{\exp \left(-\frac{G_{\mathrm{f}, i}^{\mathrm{NP}}-N_{\mathrm{Rh}, i}\left(\mu_{\mathrm{Rh}}-E_{\mathrm{Rh}}^{\text {bulk }}\right)}{k_{\mathrm{B}} T}\right)}{\sum_{k}^{N_{\mathrm{NP}}} \exp \left(-\frac{G_{\mathrm{f}, k}^{\mathrm{NP}}-N_{\mathrm{Rh}, k}\left(\mu_{\mathrm{Rh}}-E_{\mathrm{Rh}}^{\text {bulk }}\right)}{k_{\mathrm{B}} T}\right)}
$$

where the index $k$ covers all of the $N_{\mathrm{NP}}$ nanoparticles of the ensemble. Here, we assume that the nanoparticles are in thermal equilibrium with the $\mathrm{Rh}$ atoms exchanged (e.g., during the sintering process by Oswald ripening) ${ }^{49}$ which become the reservoir of the system. The chemical potential of such atoms is calculated from the conservation of the mass (total number of atoms). ${ }^{50}$ As we are not interested in simulating the dynamics of sintering, we consider the growth rates at steady state and we use a fixed value of $\mu_{\mathrm{Rh}}$. Such a value is obtained 


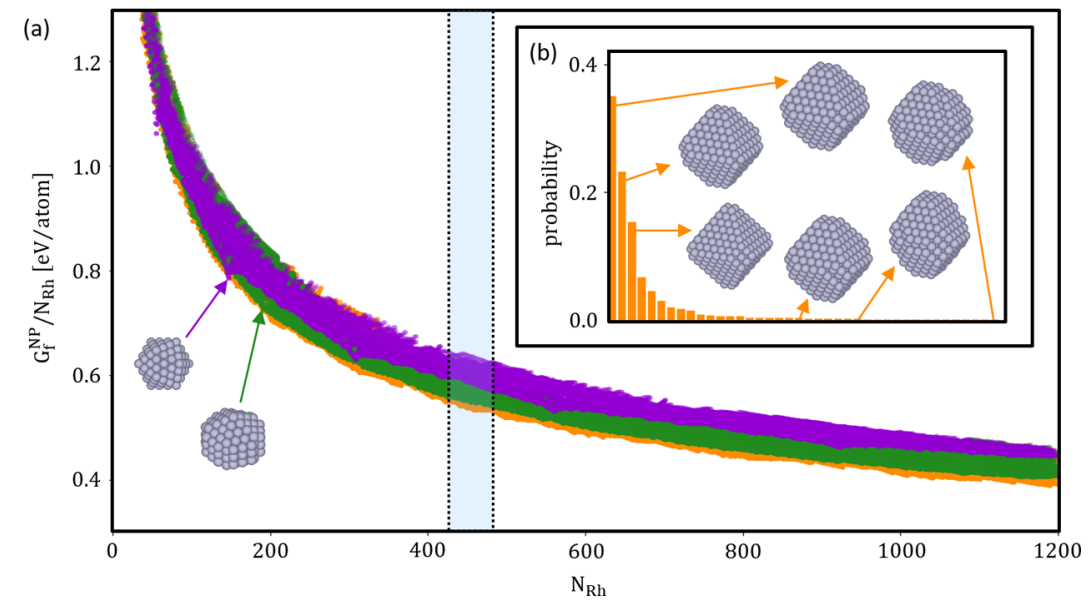

Figure 4. (a) Gibbs formation energy of the ensemble of nanoparticles with a different number of Rh atoms for fcc single crystals (in orange), decahedra (in green), and icosahedra nanoparticles (in purple). The reference for the calculation of the formation energies is set to the fcc Rh bulk. The experimental conditions are $T=823 \mathrm{~K}$ and $P_{\mathrm{CO}}=0 \mathrm{~atm}$. The nanoparticles with Gibbs formation energy per atom higher than the threshold energy function $E_{\mathrm{thr}}\left(N_{\mathrm{Rh}}\right)=0.8 E_{\mathrm{coh}}^{\text {bulk }} N_{\mathrm{Rh}}^{-1 / 3}$ are discarded. In the figure, two MTPs are also represented, stable at low $N_{\mathrm{Rh}}$ : an icosahedron with 147 $\mathrm{Rh}$ atoms and a decahedron with $192 \mathrm{Rh}$ atoms. Light blue highlights the range of number of $\mathrm{Rh}$ atoms analyzed with the Boltzmann distribution. (b) Probabilities of occurrence of the 40 most stable nanoparticles of the ensemble. Examples of nanoparticles present in the ensemble are shown as an inset in the figure.

by imposing that the total number of atoms of the ensemble equals the initial value, $N_{\text {tot }}^{\mathrm{Rh}}$

$$
\sum_{i}^{N_{\mathrm{NP}}} p_{i} N_{i}=N_{\mathrm{tot}}^{\mathrm{Rh}}
$$

As a showcase, we concentrate our analysis on the ensemble of nanoparticles with the number of atoms comprised between 420 and 480 (highlighted in Figure 4a), and we set the total number of atoms of the catalyst, $N_{\text {tot }}^{\mathrm{Rh}}$ in eq 16 , equal to the central value of the selected range ( 450 atoms). The selected range corresponds to a nanoparticle size of $2.1 \mathrm{~nm}$, the average diameter observed in experimental studies on $0.5 \% \mathrm{Rh} / \mathrm{Al}_{2} \mathrm{O}_{3}$ of Beretta et al. ${ }^{51}$ For the system analyzed, the term $\left(\mu_{\mathrm{Rh}}-\right.$ $\left.E_{\mathrm{Rh}}^{\mathrm{bulk}}\right)$ in eq 15 results $0.53 \mathrm{eV}$. The probability distribution of the ensemble of nanoparticles at $823 \mathrm{~K}$ and in the absence of $\mathrm{CO}^{*}$ is represented in Figure $4 \mathrm{~b}$. We observe that the distribution is dominated by highly symmetric $\mathrm{Rh}$ nanoparticles exposing (111) and (100) facets. However, nanoparticles with defects and high-Miller index crystal facets also show a non-negligible probability.

For the Rh catalyst sample of Beretta et al. ${ }^{51}$ that we use as an example in our analysis, after the aging of the nanoparticles at high temperature and in the presence of $\mathrm{CO}$, sintering effects were observed. The sintering process is a result of the mobility of the $\mathrm{Rh}$ catalyst atoms under reaction conditions, both in the case of nanoparticle coalescence and Ostwald ripening. ${ }^{49}$ This supports our assumption of thermal equilibrium between the nanoparticles, as, in our model, the nanoparticles are considered able to rearrange and exchange their atoms following the thermodynamic driving force.

Next, we address the quantification of the effect of the catalyst morphology on the prediction of the catalytic activity. We use as a showcase the dissociation of $\mathrm{CO}^{*}$, a structuresensitive reaction, ${ }^{52-57}$ and a relevant step, e.g., in the methanation reaction on $\mathrm{Rh}^{8}$

$$
\mathrm{CO}^{*}+* \Rightarrow \mathrm{C}^{*}+\mathrm{O}^{*}
$$

The calculation of the rate of the reaction of eq 17 at a given nanoparticle morphology needs the identification and quantification of the different types of binding sites (potential active sites) provided by the geometrical configurations of $\mathrm{Rh}$ atoms at the surface. To this aim, we compare the configurations of the surface atoms of the nanoparticle with the ones of a set of extended surfaces, for which the binding sites are known. First, we analyze the atomic configurations of the extended surfaces (considering the nearest neighbors of each surface atom) and we assign to each configuration the position of its binding sites. Then, for each $\mathrm{Rh}$ surface atom of the nanoparticle, the geometrical configuration of its nearest neighbors is compared with the ones of the set of extended surfaces. When a match is found, the binding sites of such a configuration are assigned to the nanoparticle (paying attention to avoid duplicates). In this way, we create a grid of binding sites, which link all of the surface atoms of the nanoparticle. The set of extended surfaces comprises $\mathrm{Rh}(100)$, $\mathrm{Rh}(110), \mathrm{Rh}(111), \mathrm{Rh}(311), \mathrm{Rh}(210)$, and $\mathrm{Rh}(331)$, and the types of binding sites identified are: top, bridge, the 4 -fold hollow site of (100) facet, long-bridge and long-hollow sites of (110) facet, the 3-fold hollow sites (fcc and hcp) of (111) facet, the step site $\left(B_{5}\right)$ of (311) facet, and the step site of (210) facet (which is made of two $B_{5}$ sites). An example of a grid of binding sites is given in the Supporting Information (Section 3). With such a procedure, we identify all of the binding sites for each nanoparticle in the ensemble. Then, the binding site distribution of the ensemble of nanoparticles is calculated by summing the contribution of each nanoparticle weighted by its probability

$$
N_{\alpha}^{\mathrm{POP}}=\sum_{i}^{N_{\mathrm{NP}}} N_{\alpha, i} p_{i}
$$

where $N_{\alpha, i}$ is the number of binding sites of type $\alpha$ of the nanoparticle $i$ and $N_{\alpha}^{\mathrm{POP}}$ is the resulting number of binding sites of type $\alpha$ of the ensemble.

The calculated morphology of the catalyst allowed to quantify the binding sites, which potentially can be the active sites for the reaction. To reveal the identity of the active sites that contribute relevantly to the reaction rate, the calculation of 
$\mathrm{P}_{\mathrm{CO}}=10^{-3} \mathrm{~atm}$
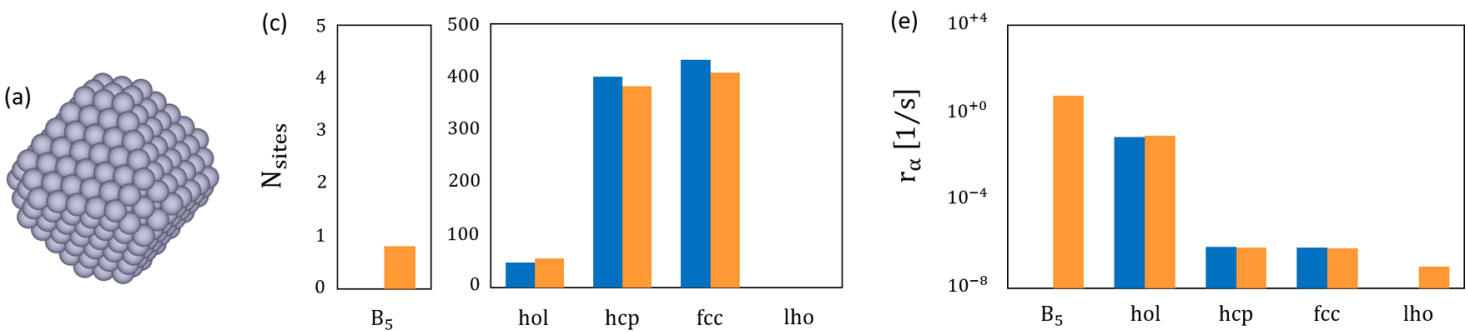

$\mathrm{P}_{\mathrm{CO}}=10^{-1} \mathrm{~atm}$
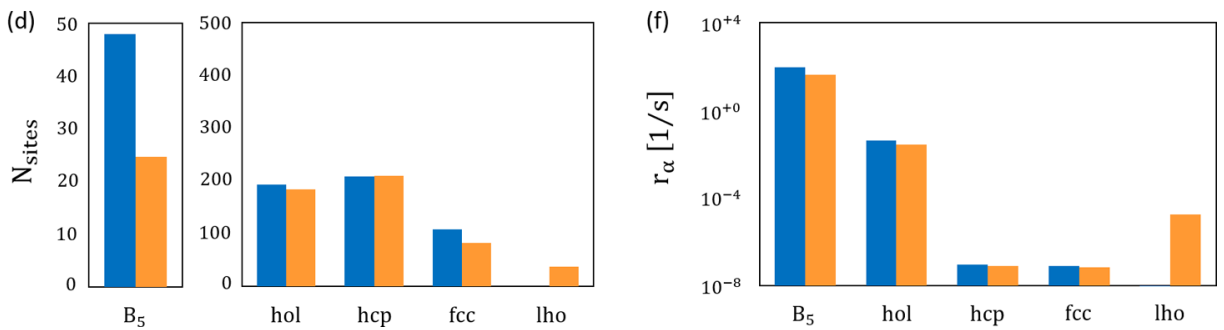

Figure 5. Ground-state shape in the ensemble at $\mathrm{CO}$ partial pressures of $10^{-3} \mathrm{~atm}(\mathrm{a})$ and $10^{-1} \mathrm{~atm}(\mathrm{~b})$. Number of selected types of active sites calculated for the ground-state shape (in blue) and for the ensemble of shapes (in orange) at CO partial pressures of $10^{-3}$ atm $(\mathrm{c})$ and $10^{-1}$ atm (d). Calculated reaction rate of $\mathrm{CO}^{*}$ dissociation provided by the different active sites at CO partial pressures of $10^{-3} \mathrm{~atm}(\mathrm{e}) \mathrm{and} 10^{-1} \mathrm{~atm}(\mathrm{f})$. Active sites considered are the $\mathrm{B}_{5}$ site, typical of (311), (211), and (210) facets; fcc and hcp provided by the (111) facets; the hollow (hol) site of the (100) facet; the long-hollow (lho) site, typical of the (110) facet. The data reported are per particle for a sample with an average number of Rh atoms equal to 450 .

the catalytic activity of these sites is needed. To this aim, by simulating the reaction paths with nudged elastic band $(\mathrm{NEB})^{30}$ calculations and applying harmonic transition state theory (h-TST), we calculate the kinetic parameters of the $\mathrm{CO}^{*}$ dissociation (eq 17) on all of the sites of the considered extended surfaces. In agreement with previous studies, ${ }^{7,8,58}$ we find that the lowest Gibbs activation energy is provided by the $\mathrm{B}_{5}$ sites of $\mathrm{Rh}(311)$ and $\mathrm{Rh}(210)$ facets. The active site with the second higher catalytic activity is found to be the hollow site of $\mathrm{Rh}(100)$. Other active sites of the reaction are the longhollow of $\mathrm{Rh}(110)$ and fcc and hcp of $\mathrm{Rh}(111)$. It has to be pointed out that the coordination environment of the atoms that provide the active sites can have an impact on the Gibbs activation energy. This is a particularly important aspect to take into account if we want to use the kinetic parameters obtained from calculations on extended surfaces to calculate the reaction rates on the active sites of the nanoparticles. Indeed, due to their finite size, the surfaces exposed by the nanoparticles can be made of just a few atoms and therefore exhibit different coordination environments compared to the respective periodic surfaces. For $\mathrm{CO}^{*}$ dissociation, however, we calculate that the $B_{5}$ sites provided by $R$ h crystal facets with different coordination environments show similar activation Gibbs free energies $(1.7-1.8 \mathrm{eV})$, which are significantly lower than the other active sites $(2.2-3.1 \mathrm{eV})$. The data are reported in Table S4. Given this strong dependence of the reactivity on the type of site, in our analysis, we neglect the effect of the detailed coordination environment. This allows us, for the specific case of the $\mathrm{CO}^{*}$ dissociation, to use the kinetic parameters obtained from periodic surfaces to describe the catalytic activity of the nanoparticles' active sites. Details on the calculation of the kinetic parameters of $\mathrm{CO}^{*}$ dissociation on $\mathrm{Rh}$ facets are provided in the Supporting Information (Section 3).
For each site $\alpha$ present in the distribution of the active sites, the reaction rate, $r_{\alpha}$, is calculated as

$$
r_{\alpha}=\frac{k_{\mathrm{B}} T}{h_{\mathrm{P}}} \exp \left(-\frac{G_{\mathrm{act}, \alpha}}{k_{\mathrm{B}} T}\right) \vartheta_{\mathrm{CO} *}\left(1-\vartheta_{\mathrm{CO} *}\right)
$$

where $h_{\mathrm{P}}$ is the Planck constant, $\vartheta_{\mathrm{CO}}$ is the $\mathrm{CO}$ coverage on the nanoparticle, and $G_{\mathrm{act}, \alpha}$ is the Gibbs activation energy of the elementary step on the site $\alpha$. The mean field approximation is employed in eq 19 , as our calculations show that the activation energy of the $\mathrm{CO}^{*}$ surface diffusion (about $0.12 \mathrm{eV}$ ) is much lower than the activation energy of $\mathrm{CO}^{*}$ dissociation (higher than $1.7 \mathrm{eV}$ ).

The total reaction rate, $r_{\text {tot }}$ is calculated as the sum of the rates of each active site multiplied by its number

$$
r_{\text {tot }}=\sum_{\alpha}^{N_{\text {sites }}} r_{\alpha} N_{\alpha}
$$

where $N_{\text {sites }}$ is the total number of types of active sites.

We analyze the ensemble of nanoparticles represented in Figure $4 \mathrm{~b}$ (with number of atoms comprised between 420 and 480 ) at $823 \mathrm{~K}$ and at two different partial pressures of $\mathrm{CO}$ in the gas phase: $10^{-3}$ and $10^{-1} \mathrm{~atm}$. To study the effect of the presence of metastable structures on the catalytic activity, we compare the ensemble of nanoparticles obtained by Boltzmann statistics (eq 15) with a corresponding sample (with the same number of atoms) made of nanoparticles in the most stable shape (the ground-state shape), which can be obtained by filling a Wulff construction with $\mathrm{Rh}$ atoms. The Gibbs formation energies and the corresponding probability distribution of the nanoparticles in the ensemble at low and high CO partial pressures are represented in Figure S12.

At diluted conditions $\left(P_{\mathrm{CO}}=10^{-3} \mathrm{~atm}\right)$, the nanoparticles are almost clean: the $\mathrm{CO}^{*}$ coverage on all of the stable 
nanoparticles is very low (below $0.05 \mathrm{ML}$ ); therefore, its effect on the energy and the distribution of the nanoparticles is negligible. The ground-state shape in these conditions is demonstrated in Figure 5a. The distributions of active sites of the ground-state shape and of the ensemble of nanoparticles are shown in Figure 5b. Between the two active sites' distributions, we can notice some differences. Importantly, the ensemble of shapes shows a non-negligible number of $B_{5}$ sites, which are not revealed by the ground-state shape.

The higher $P_{\mathrm{CO}}$ in the system $\left(10^{-1} \mathrm{~atm}\right)$ induces drastic changes in the catalyst morphology and in the active sites' distribution. The ground-state shape becomes a spherical nanoparticle (shown in Figure 5b) exposing (110) and (311) facets with a coverage of $0.84 \mathrm{ML}$, higher than the average coverage of the ensemble $(0.79 \mathrm{ML})$. At these conditions, the number of $\mathrm{B}_{5}$ and long-hollow sites is enhanced, at the expenses of fcc and hcp sites, for both ground-state shape and ensemble of shapes (Figure 5d). When we compare the active sites' distribution of the ground-state shape and the ensemble of nanoparticles, we notice that the ground-state shape shows a significantly higher number of $\mathrm{B}_{5}$ sites. The transformation of $\mathrm{Rh}$ particles to spherical shapes in the presence of adsorbates at high coverage is consistent with the calculations of Mittendorfer et al. ${ }^{59}$ based on $a b$ initio thermodynamics.

For $P_{\mathrm{CO}}=10^{-3} \mathrm{~atm}$, when we calculate the total reaction rate provided by all of the active sites of the ground-state shape (with eq 20), we obtain a value of $0.08 \mathrm{~s}^{-1}\left(7.7 \times 10^{-4} \mathrm{kmol}\right.$ $\left.\mathrm{kg}_{\mathrm{Rh}^{-1}} \mathrm{~s}^{-1}\right)$. The rate turns out to be 2 orders of magnitude higher, $6.20 \mathrm{~s}^{-1}\left(6.0 \times 10^{-2} \mathrm{kmol} \mathrm{kg}_{\mathrm{Rh}}^{-1} \mathrm{~s}^{-1}\right)$, when we account for the ensemble of nanoparticles by means of eq 15 . Indeed, the reaction rate strongly depends on the quantification of the amount of $B_{5}$ sites of the catalyst, which dominate the reaction rate (Figure $5 \mathrm{e}$ ) and are absent in the ground-state shape. As a consequence, the ensemble of shapes shows a much higher catalytic activity.

At $P_{\mathrm{CO}}=10^{-1} \mathrm{~atm}$, we calculate a total reaction rate of 80.80 $\mathrm{s}^{-1}\left(7.9 \times 10^{-1} \mathrm{kmol} \mathrm{kg}_{\mathrm{Rh}}^{-1} \mathrm{~s}^{-1}\right)$ for the ground-state shape. Significantly lower is the one calculated for the ensemble of shapes: $35.90 \mathrm{~s}^{-1}\left(3.5 \times 10^{-1} \mathrm{kmol} \mathrm{kg}_{\mathrm{Rh}}{ }^{-1} \mathrm{~s}^{-1}\right)$. The $\mathrm{B}_{5}$ sites also control the reaction rate in these systems (Figure 5f), and their quantification changes significantly when we consider the presence of metastable nanoparticles in the catalyst ensemble of shapes, reflecting in a considerably different calculated reaction rate.

For the $\mathrm{CO}^{*}$ dissociation elementary step on $\mathrm{Rh}$ nanoparticles, we found that at low $\mathrm{CO}^{*}$ coverage, the most active sites are provided by configurations of atoms absent in the ground-state shape, and the rate of the reaction is enhanced drastically by the high-energy nanoparticles present in the ensemble. If we neglect the presence of metastable structures in the modeling of the catalyst structure, we severely underestimate the catalytic activity and we fail in the identification of the active site that governs the chemical transformation. At high coverage, the presence of adsorbates induces important morphological transformations of the catalyst, and the ground-state shape has a higher amount of the most active sites compared to the ensemble of nanoparticles. As a result, if we model the catalyst morphology considering only the ground-state shape, we introduce an error in the calculation of the catalytic activity. Our findings highlight the importance of modeling nanocatalyst morphology based on ensembles of nanoparticles for the determination of structure-sensitive reaction rates in heterogeneous catalysis.
In principle, these results may depend on the accuracy of the thermodynamic model employed. However, the probabilities of the nanoparticles calculated with the Boltzmann distribution (eq 15) are determined by the differences in the Gibbs formation energies of the nanoparticles, and most of the errors arising from the approximations of the model are expected to cancel out when we evaluate those energy differences. This is particularly true when the nanoparticles have similar morphologies and different catalytic activities due to the difference in their distribution of active sites (e.g., for the case at low $\mathrm{CO}^{*}$ coverage).

The presented results do not include the effect of the interface between the nanoparticles and the support material on which they can be supported. However, our methodology can be potentially extended to account for the presence of the support by taking into consideration the formation energy of metal atoms in contact with the support. ${ }^{60,61}$ The main advantage of the proposed methodology consists in its versatility. The sampling of the configurational space performed in the production of the ensemble of nanoparticle shapes is separated from the calculation of the energy of the resulting structures. Consequently, the ensemble of shapes produced with the methodology herein can also be employed for the analysis of supported systems, as well as of nanoparticles of different metals and in other reaction environments, once a thermodynamic model is developed for the calculation of their formation energies.

The library of scripts employed in this work is provided in the Supporting information. The latest version of the source code is available at the Github page: github.com/ raffaelecheula/nanoparticles_ensembles.

\section{CONCLUSIONS}

In this work, we introduce a methodology to simulate reaction rates in heterogeneous catalysis that originate from ensembles of nanoparticles under experimental conditions. First, we presented a procedure for the creation of an ensemble of nanoparticles representative of the diversity of shapes observed experimentally. The ensemble contains single crystals and multiply-twinned particles with both high and low symmetries and complete or incomplete crystal facets. Then, we formulated and applied a model for the calculation of the Gibbs free energy of Rh nanoparticles of any size and shape. The model takes into consideration the effect of adsorbed CO molecules on the energy of the nanoparticles. We employed a Boltzmann distribution to calculate the probability of each nanoparticle based on the calculated Gibbs formation energy, and we addressed the effect of metastable nanostructures on the overall catalytic activity. In particular, we analyzed the CO* dissociation elementary step and demonstrated that the contribution of metastable nanoparticles to the reaction rate can be drastic due to providing the necessary active sites. Importantly, we demonstrated that the number of the most active sites can change drastically when we analyze the groundstate shape vs the ensemble of shapes, which in turn can dramatically impact the overall calculated reaction rates.

\section{ASSOCIATED CONTENT}

\section{Supporting Information}

The Supporting Information is available free of charge at https://pubs.acs.org/doi/10.1021/acscatal.0c01005. 
Creation of the ensemble of nanoparticles (Section 1); model for Gibbs free energy of formation of $\mathrm{Rh}$ nanoparticles in the presence of CO (Section 2); identification of active sites and calculation of reaction rates of $\mathrm{CO}^{*}$ dissociation (Section 3) (PDF)

Source code of the library of Python and Cython scripts employed in the work (ZIP)

\section{AUTHOR INFORMATION}

\section{Corresponding Authors}

Matteo Maestri - Laboratory of Catalysis and Catalytic Processes, Dipartimento di Energia, Politecnico di Milano, 20156 Milano, Italy; 이이.org/0000-0002-8925-3869; Email: matteo.maestri@polimi.it

Giannis Mpourmpakis - Department of Chemical and Petroleum Engineering, University of Pittsburgh, Pittsburgh, Pennsylvania 15260, United States; 이이. orcid.org/0000-00023063-0607; Email: gmpourmp@pitt.edu

\section{Author}

Raffaele Cheula - Laboratory of Catalysis and Catalytic Processes, Dipartimento di Energia, Politecnico di Milano, 20156 Milano, Italy; Department of Chemical and Petroleum Engineering, University of Pittsburgh, Pittsburgh, Pennsylvania 15260, United States

Complete contact information is available at: https://pubs.acs.org/10.1021/acscatal.0c01005

\section{Author Contributions}

The manuscript was written through the contributions of all authors. All authors have given approval to the final version of the manuscript.

\section{Notes}

The authors declare no competing financial interest.

\section{ACKNOWLEDGMENTS}

M.M. and R.C. acknowledge support by the European Research Council (ERC) under the European Union's Horizon 2020 research and innovation programme (Project SHAPE, grant agreement No. 677423). G.M. acknowledges support by the National Science Foundation under Grant 1634880 (CMMI). R.C. gratefully acknowledges a scholarship by the Foundation Blanceflor Boncompagni Ludovisi, née Bildt. Computational support was provided by the Center for Research Computing at the University of Pittsburgh and CINECA, Bologna (Italy).

\section{REFERENCES}

(1) Schlögl, R. Heterogeneous Catalysis. Angew. Chem., Int. Ed. 2015, 54, 3465-3520.

(2) Sabbe, M. K.; Reyniers, M. F.; Reuter, K. First-Principles Kinetic Modeling in Heterogeneous Catalysis: An Industrial Perspective on Best-Practice, Gaps and Needs. Catal. Sci. Technol. 2012, 2, 20102024.

(3) Mpourmpakis, G.; Andriotis, A. N.; Vlachos, D. G. Identification of Descriptors for the CO Interaction with Metal Nanoparticles. Nano Lett. 2010, 10, 1041-1045.

(4) Dean, J.; Taylor, M. G.; Mpourmpakis, G. Unfolding Adsorption on Metal Nanoparticles: Connecting Stability with Catalysis. Sci. Adv. 2019, 5, No. eaax5101.

(5) Van Santen, R. A. Complementary Structure Sensitive and Insensitive Catalytic Relationships. Acc. Chem. Res. 2009, 42, 57-66.
(6) Honkala, K.; Hellman, A.; Remediakis, I. N.; Logadottir, A.; Carlsson, A.; Dahl, S.; Christensen, C. H.; Nørskov, J. K. Ammonia Synthesis from First-Principles Calculations. Science 2005, 307, 555558.

(7) Filot, I. A. W.; Shetty, S. G.; Hensen, E. J. M.; Van Santen, R. A. Size and Topological Effects of Rhodium Surfaces, Clusters and Nanoparticles on the Dissociation of CO. J. Phys. Chem. C 2011, 115, 14204-14212.

(8) Mavrikakis, M.; Bäumer, M.; Freund, H. J.; Nørskov, J. K. Structure Sensitivity of CO Dissociation on Rh Surfaces. Catal. Lett. 2002, 81, 153-156.

(9) Austin, N.; Johnson, J. K.; Mpourmpakis, G. $A_{13}$ : CO Adsorbs, Nanoparticle Responds. J. Phys. Chem. C 2015, 119, 18196-18202.

(10) Taylor, M. G.; Austin, N.; Gounaris, C. E.; Mpourmpakis, G. Catalyst Design Based on Morphology- and Environment-Dependent Adsorption on Metal Nanoparticles. ACS Catal. 2015, 5, 6296-6301.

(11) Avanesian, T.; Dai, S.; Kale, M. J.; Graham, G. W.; Pan, X.; Christopher, P. Quantitative and Atomic-Scale View of CO-Induced Pt Nanoparticle Surface Reconstruction at Saturation Coverage via DFT Calculations Coupled with in Situ TEM and IR. J. Am. Chem. Soc. 2017, 139, 4551-4558.

(12) Cheula, R.; Soon, A.; Maestri, M. Prediction of Morphological Changes of Catalyst Materials under Reaction Conditions by Combined $\mathrm{Ab}$ Initio Thermodynamics and Microkinetic Modelling. Catal. Sci. Technol. 2018, 8, 3493-3503.

(13) Marks, L. D.; Peng, L. Nanoparticle Shape, Thermodynamics and Kinetics. J. Phys.: Condens. Matter 2016, 28, No. 053001.

(14) Soon, A.; Todorova, M.; Delley, B.; Stampfl, C. Thermodynamic Stability and Structure of Copper Oxide Surfaces: A FirstPrinciples Investigation. Phys. Rev. B 2007, 75, No. 125420.

(15) Richter, N. A.; Kim, C.-E.; Stampfl, C.; Soon, A. Re-Visiting the $\mathrm{O} / \mathrm{Cu}(111)$ System - When Metastable Surface Oxides Could Become an Issue! Phys. Chem. Chem. Phys. 2014, 16, 26735-26740.

(16) Hörmann, N. G.; Andreussi, O.; Marzari, N. Grand Canonical Simulations of Electrochemical Interfaces in Implicit Solvation Models. J. Chem. Phys. 2019, 150, No. 041730.

(17) Bonnet, N.; Marzari, N. First-Principles Prediction of the Equilibrium Shape of Nanoparticles under Realistic Electrochemical Conditions. Phys. Rev. Lett. 2013, 110, No. 086104.

(18) Pohl, D.; Surrey, A.; Schultz, L.; Rellinghaus, B. The Impact of Oxygen on the Morphology of Gas-Phase Prepared Au Nanoparticles. Appl. Phys. Lett. 2012, 101, No. 263105.

(19) Copper, S. F. T.; Silly, F.; Castell, M. R. TemperatureDependent Stability of Supported Five-Fold Twinned Copper Nanocrystals. ACS Nano 2009, 3, 901-906.

(20) Barnard, A. S. Modelling of Nanoparticles: Approaches to Morphology and Evolution. Rep. Prog. Phys. 2010, 73, No. 086502.

(21) Ha, M. A.; Dadras, J.; Alexandrova, A. Rutile-Deposited Pt-Pd Clusters: A Hypothesis Regarding the Stability at 50/50 Ratio. ACS Catal. 2014, 4, 3570-3580.

(22) Baxter, E. T.; Ha, M. A.; Cass, A. C.; Alexandrova, A. N.; Anderson, S. L. Ethylene Dehydrogenation on $\mathrm{Pt}_{4,7,8}$ Clusters on $\mathrm{Al}_{2} \mathrm{O}_{3}$ : Strong Cluster Size Dependence Linked to Preferred Catalyst Morphologies. ACS Catal. 2017, 7, 3322-3335.

(23) Sun, G.; Sautet, P. Metastable Structures in Cluster Catalysis from First-Principles: Structural Ensemble in Reaction Conditions and Metastability Triggered Reactivity. J. Am. Chem. Soc. 2018, 140, 2812-2820.

(24) Barnard, A. S. Clarifying Stability, Probability and Population in Nanoparticle Ensembles. Nanoscale 2014, 6, 9983.

(25) Rahm, J. M.; Erhart, P. Beyond Magic Numbers: Atomic Scale Equilibrium Nanoparticle Shapes for Any Size. Nano Lett. 2017, 17, 5775-5781.

(26) Giannozzi, P.; Baroni, S.; Bonini, N.; Calandra, M.; Car, R.; Cavazzoni, C.; Ceresoli, D.; Chiarotti, G. L.; Cococcioni, M.; Dabo, I.; Dal Corso, A.; de Gironcoli, S.; Fabris, S.; Fratesi, G.; Gebauer, R.; Gerstmann, U.; Gougoussis, C.; Kokalj, A.; Lazzeri, M.; MartinSamos, L.; Marzari, N.; Mauri, F.; Mazzarello, R.; Paolini, S.; Pasquarello, A.; Paulatto, L.; Sbraccia, C.; Scandolo, S.; Sclauzero, G.; 
Seitsonen, A. P.; Smogunov, A.; Umari, P.; Wentzcovitch, R. M. QUANTUM ESPRESSO: A Modular and Open-Source Software Project for Quantum Simulations of Materials. J. Phys.: Condens. Matter 2009, 21, No. 395502.

(27) Perdew, J. P.; Burke, K.; Ernzerhof, M. Generalized Gradient Approximation Made Simple. Phys. Rev. Lett. 1996, 77, 3865-3868.

(28) Monkhorst, H. J.; Pack, J. D. Special Points for Brillouin-Zone Integrations. Phys. Rev. B 1976, 13, 5188-5192.

(29) Marzari, N.; Vanderbilt, D.; De Vita, A.; Payne, M. C. Thermal Contraction and Disordering of the $\mathrm{Al}(110)$ Surface. Phys. Rev. Lett. 1999, 82, 3296-3299.

(30) Henkelman, G.; Uberuaga, B. P.; Jónsson, H. A Climbing Image Nudged Elastic Band Method for Finding Saddle Points and Minimum Energy Paths. J. Chem. Phys. 2000, 113, 9901-9904.

(31) Hjorth Larsen, A.; Jørgen Mortensen, J.; Blomqvist, J.; Castelli, I. E.; Christensen, R.; Dulak, M.; Friis, J.; Groves, M. N.; Hammer, B.; Hargus, C.; Hermes, E. D.; Jennings, P. C.; Bjerre Jensen, P.; Kermode, J.; Kitchin, J. R.; Leonhard Kolsbjerg, E.; Kubal, J.; Kaasbjerg, K.; Lysgaard, S.; Bergmann Maronsson, J.; Maxson, T.; Olsen, T.; Pastewka, L.; Peterson, A.; Rostgaard, C.; SchiØtz, J.; Schütt, O.; Strange, M.; Thygesen, K. S.; Vegge, T.; Vilhelmsen, L.; Walter, M.; Zeng, Z.; Jacobsen, K. W. The Atomic Simulation Environment - A Python Library for Working with Atoms. J. Phys.: Condens. Matter 2017, 29, No. 273002.

(32) Behnel, S.; Bradshaw, R.; Citro, C.; Dalcin, L.; Seljebotn, D. S.; Smith, K. Cython: The Best of Both Worlds. Comput. Sci. Eng. 2011, 13, 31-39.

(33) Tománek, D.; Mukherjee, S.; Bennemann, K. H. Simple Theory for the Electronic and Atomic Structure of Small Clusters. Phys. Rev. B 1983, 28, 665-673.

(34) Yan, Z.; Taylor, M. G.; Mascareno, A.; Mpourmpakis, G. Size-, Shape-, and Composition-Dependent Model for Metal Nanoparticle Stability Prediction. Nano Lett. 2018, 18, 2696-2704.

(35) Sun, C. Q.; Tay, B. K.; Zeng, X. T.; Li, S.; Chen, T. P.; Zhou, J.; Bai, H. L.; Jiang, E. Y. Bond-Order-Bond-Length-Bond-Strength (Bond-OLS) Correlation Mechanism for the Shape-and-Size Dependence of a Nanosolid. J. Phys.: Condens. Matter 2002, 14, 7781-7795.

(36) Howie, A.; Marks, L. D. Elastic Strains and the Energy Balance for Multiply Twinned Particles. Philos. Mag. A 1984, 49, 95-109.

(37) Donazzi, A.; Beretta, A.; Groppi, G.; Forzatti, P. Catalytic Partial Oxidation of Methane over a $4 \% \mathrm{Rh} / \mathrm{Alpha}-\mathrm{Al}_{2} \mathrm{O}_{3}$ Catalyst Part II: Role Of $\mathrm{CO}_{2}$ Reforming. J. Catal. 2008, 255, 259-268.

(38) Schädel, B. T.; Duisberg, M.; Deutschmann, O. Steam Reforming of Methane, Ethane, Propane, Butane, and Natural Gas over a Rhodium-Based Catalyst. Catal. Today 2009, 142, 42-51.

(39) Strisland, F.; Ramstad, A.; Ramsvik, T.; Borg, A. CO Adsorption on the $\mathrm{Rh}(100)$ Surface Studied by High Resolution Photoelectron Spectroscopy. Surf. Sci. 1998, 415, L1020-L1026.

(40) Linke, R.; Curulla, D.; Hopstaken, M. J. P.; Niemantsverdriet, J. W. $\mathrm{CO} / \mathrm{Rh}(111)$ : Vibrational Frequency Shifts and Lateral Interactions in Adsorbate Layers. J. Chem. Phys. 2001, 115, 82098216.

(41) Dhanak, V. R.; Baraldi, A.; Comelli, G.; Paolucci, G.; Kiskinova, M.; Rosei, R. CO Adsorption on Unreconstructed and Reconstructed Rh(110) Surfaces: LEED and XPS Studies. Surf. Sci. 1993, 295, 287294.

(42) Gurney, B. A.; Richter, L. J.; Villarrubia, J. S.; Ho, W. The Populations of Bridge and Top Site $\mathrm{CO}$ on $\mathrm{Rh}(100)$ vs Coverage, Temperature, and during Reaction with O. J. Chem. Phys. 1987, 87, $6710-6721$

(43) Gierer, M.; Barbieri, A.; Van Hove, M. A.; Somorjai, G. A. Structural Reanalysis of the $\mathrm{Rh}(111)+(\mathrm{V} 3 \times \mathrm{V} 3) \mathrm{R} 30^{\circ}-\mathrm{CO}$ and $\mathrm{Rh}(111)+(2 \times 2)-3 \mathrm{CO}$ Phases Using Automated Tensor LEED. Surf. Sci. 1997, 391, 176-182.

(44) Mason, S. E.; Grinberg, I.; Rappe, A. M. First-Principles Extrapolation Method for Accurate CO Adsorption Energies on Metal Surfaces. Phys. Rev. B 2004, 69, No. 161401.

(45) Rogal, J.; Reuter, K. Ab Initio Atomistic Thermodynamics for Surfaces: A Primer. In Experiment, Modeling and Simulation of Gas-
Surface Interactions for Reactive Flows in Hypersonic Flights; RTO, 2007; pp 2-1-2-18.

(46) Sprowl, L. H.; Campbell, C. T.; Árnadóttir, L. Hindered Translator and Hindered Rotor Models for Adsorbates: Partition Functions and Entropies. J. Phys. Chem. C 2016, 120, 9719-9731.

(47) Mcbride, B.; Gordon, S.; Reno, M. Coefficients for Calculating Thermodynamic and Transport Properties of Individual Species, NASA Technical Memorandum TM-4513; NASA, 1993; Vol. 4513, p 98.

(48) Garden, A. L.; Pedersen, A.; Jónsson, H. Reassignment of "magic Numbers" for Au Clusters of Decahedral and FCC Structural Motifs. Nanoscale 2018, 10, 5124-5132.

(49) Campbell, C. T.; Mao, Z. Chemical Potential of Metal Atoms in Supported Nanoparticles: Dependence upon Particle Size and Support. ACS Catal. 2017, 7, 8460-8466.

(50) Houk, L. R.; Challa, S. R.; Grayson, B.; Fanson, P.; Datye, A. K. The Definition of "Critical Radius" for a Collection of Nanoparticles Undergoing Ostwald Ripening. Langmuir 2009, 25, 11225-11227.

(51) Beretta, A.; Donazzi, A.; Groppi, G.; Forzatti, P.; Dal Santo, V.; Sordelli, L.; De Grandi, V.; Psaro, R. Testing in Annular MicroReactor and Characterization of Supported $\mathrm{Rh}$ Nanoparticles for the Catalytic Partial Oxidation of Methane: Effect of the Preparation Procedure. Appl. Catal., B 2008, 83, 96-109.

(52) Andersson, S.; Frank, M.; Sandell, A.; Libuda, J.; Brena, B.; Giertz, A.; Brühwiler, P. A.; Bäumer, M.; Mårtensson, N.; Freund, H. J. Temperature Dependent XPS Study of CO Dissociation on Small Rh Particles. Vacuum 1998, 49, 167-170.

(53) Andersson, S.; Frank, M.; Sandell, A.; Giertz, A.; Brena, B.; Brühwiler, P. A.; Mårtensson, N.; Libuda, J.; Baümer, M.; Freund, H. J. CO Dissociation Characteristics on Size-Distributed Rhodium Islands on Alumina Model Substrates. J. Chem. Phys. 1998, 108, 2967-2974.

(54) Frank, M.; Andersson, S.; Libuda, J.; Stempel, S.; Sandell, A.; Brena, B.; Giertz, A.; Brühwiler, P. A.; Bäumer, M.; Mårtensson, N.; Freund, H. J. Particle Size Dependent CO Dissociation on AluminaSupported Rh: A Model Study. Chem. Phys. Lett. 1997, 279, 92-99.

(55) Cho, B. K.; Stock, C. J. Dissociation and Oxidation of Carbon Monoxide over $\mathrm{Rh} / \mathrm{Al}_{2} \mathrm{O}_{3}$ Catalysts. J. Catal. 1989, 117, 202-217.

(56) Matolín, V.; Elyakhloufi, M. H.; Mašek, K.; Gillet, E. CO Dissociation and Oxidation on Small Supported Rhodium Particles: SSIMS and TPR Study. Catal. Lett. 1993, 21, 175-182.

(57) Nehasil, V.; Stará, I.; Matolín, V. Study of CO Desorption and Dissociation on Rh Surfaces. Surf. Sci. 1995, 331-333, 105-109.

(58) Liu, Z. P.; Hu, P. General Rules for Predicting Where a Catalytic Reaction Should Occur on Metal Surfaces: A Density Functional Theory Study of C-H and C-O Bond Breaking/Making on Flat, Stepped, and Kinked Metal Surfaces. J. Am. Chem. Soc. 2003, $125,1958-1967$.

(59) Mittendorfer, F.; Seriani, N.; Dubay, O.; Kresse, G. Morphology of Mesoscopic Rh and Pd Nanoparticles under Oxidizing Conditions. Phys. Rev. B 2007, 76, No. 233413.

(60) Tan, K.; Dixit, M.; Dean, J.; Mpourmpakis, G. Predicting Metal-Support Interactions in Oxide-Supported Single-Atom Catalysts. Ind. Eng. Chem. Res. 2019, 58, 20236-20246.

(61) Hemmingson, S. L.; Campbell, C. T. Trends in Adhesion Energies of Metal Nanoparticles on Oxide Surfaces: Understanding Support Effects in Catalysis and Nanotechnology. ACS Nano 2017, 11, 1196-1203. 